\title{
Continuum model of acoustic metamaterials with diatomic crystal lattice
}

\section{Yahong Zhou, Peijun Wei, Yueqiu Li \& Qiheng Tang}

To cite this article: Yahong Zhou, Peijun Wei, Yueqiu Li \& Qiheng Tang (2016): Continuum model of acoustic metamaterials with diatomic crystal lattice, Mechanics of Advanced Materials and Structures, DOI: 10.1080/15376494.2016.1205685

To link to this article: http://dx.doi.org/10.1080/15376494.2016.1205685

Accepted author version posted online: 27

Jun 2016.

Published online: 27 Jun 2016.

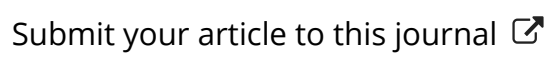

View related articles ¿

View Crossmark data ¿ 


\title{
ACCEPTED MANUSCRIPT
}

\section{Continuum model of acoustic metamaterials with diatomic crystal lattice}

\author{
Yahong Zhou ${ }^{1}$, Peijun Wei ${ }^{1,2, *}$, Yueqiu $\mathrm{Li}^{1}$, and Qiheng Tang ${ }^{2}$ \\ ${ }^{1}$ Department of Applied Mechanics, University of Sciences and Technology Beijing, Beijing, \\ China \\ ${ }^{2}$ State Key Laboratory of Nonlinear Mechanics (LNM), Chinese Academy of Science, Beijing, \\ China \\ *CONTACT Peijun Wei, weipj@ustb.edu.cn, Department of Applied Mechanics, University of \\ Sciences and Technology Beijing, Beijing 100083, China. \\ Color versions of one or more figures in this article are available online at \\ www.tandfonline.com/umcm.
}

\begin{abstract}
The continuum model of one-dimensional acoustic metamaterial with diatomic crystal lattice is studied in this article. First, the dispersive relation of lattice wave in one-dimensional diatomic crystal lattice of metamaterial is established and compared with that of the classic material. Then, the continuum model of the acoustic metamaterial leads to the classical continuum model, the stain gradient continuum model and the nonlocal gradient continuum model based on different assumptions. The dispersive curves which correspond to the three kinds of models are shown graphically and compared with that of discrete crystal lattice of metamaterial. The disadvantage of the classic continuum model and the strain gradient continuum model are discussed. The
\end{abstract}




\section{ACCEPTED MANUSCRIPT}

nonlocal gradient continuum model is derived based on the nonlocal assumption of continuous displacement field. The stability of dispersive curves is guaranteed. However, the actual prediction effects are still dependent upon the appropriate selection of the nonlocal parameter in the nonlocal gradient continuum model.

\section{Keywords}

Metamaterial, Dispersion relations, diatomic lattice, strain gradient continuum, nonlocal gradient continuum 


\section{ACCEPTED MANUSCRIPT}

\section{Introduction}

Recently, many researchers were engaged in the exploring of the acoustic metamaterial, e.g. Liu and Zhang et al (2000), Pendry (2000), Li and Chan (2004), Fang and Xi (2006), Sheng and Mei et al (2007), Lee and Park et al (2009), Lu and Fang et al (2009), Kadic and Buckmann et al (2012). The so called acoustic metamaterial are generally regarded as materials that possess negative effective mass or negative effective modulus, which is similar with the electromagnetic metamaterials that exhibit negative electric permittivity $(\varepsilon)$, negative magnetic permeability $(\mu)$, and hence a negative refractive index. The unusual properties of acoustic metamaterial can make the acoustic wave beam bending and getting round the objective to be concealed. The potential application on the designing acoustic cloak inspired the research interests on the acoustic metamaterial. The existing researches showed that the unusual properties of acoustic metamaterial, which are not readily observed in natural materials, result from the manmade microstructures that embedded in host material. These manmade microstructures play a role of the mechanical resonators and create unusual properties mainly based on the local resonance of resonator. In order to study the mechanical behavior of acoustic metamaterial, Milton and Willis (2007) proposed the mass-in-mass discrete model of acoustic metamaterial. Their study showed that the effective mass should be represented by a second order tensor and can be anisotropic and frequency-dependent. Huang, Sun and Huang (2009) studied the dispersive wave propagation in the mass-in-mass lattice system and compared to various equivalent models. It is found that, if 


\section{ACCEPTED MANUSCRIPT}

the classical elastic continuum is used to represent the original mass-in-mass lattice system, the effective mass density becomes frequency dependent and may become negative for frequencies near the resonance frequency of the internal mass. Huang and Sun (2009) studied the wave attenuation and energy transfer mechanisms of a metamaterial having a negative effective mass density based on the mass-in-mass system. It is found that most of the work done by the external force on the lattice system is stored by the internal mass if the forcing frequency is close to the local resonance frequency. Huang and Sun (2010) further investigated the multi-resonator mass-in-mass lattice system. The unit cell of the lattice system consists of three separate masses connected by linear springs.

The acoustic metamaterials can also be modeled as classical homogeneous solids with periodically distributed microstructure. A continuum model for the acoustic metamaterial with the microstructure effect taken into consideration is therefore desired. Mindlin (1964) established early a linear elastic theory of solid with microstructure where macromotion and micromotion coexist and showed that there are four micro-vibration modes. The coupling between the macro-vibration and the micro-vibration leads to twelve kinds of wave modes. Eight of them are the dispersive. Too many motion freedoms and material parameters make the application of the microstructure elastic theories restricted. In contrast, the gradient elastic theory has less material parameters and therefore attracted wide attentions in the last two decades. Askes and Aifantis (2011) gave a comprehensive and detailed review of various gradient elasticity formats including 


\section{ACCEPTED MANUSCRIPT}

the multi-scale format and the mono-scale format, the stress gradient format and the strain gradient format, the stable strain gradient format and the unstable strain gradient format and so on. Metrikine and Askes (2002) also presented a gradient elasticity model derived from a discrete microstructure. In their work, a new continuation method is proposed in which each higher-order stiffness term is accompanied by a higher order inertia term. As such, the resulting models are dynamically consistent. Because physically realistic behavior is obtained in statics and dynamics, their gradient elasticity models is superior to earlier gradient elasticity models in which there are not anomalies in the dynamic behavior. Milton and Willis (2007) investigated the macroscopic behavior of an elastic metamaterials. The microstructure effects are taken into consideration by the modification of linear elastodynamic equations. The total stress is not only related with strain but also with velocity, and the momentum density is not only related with velocity but also with the displacement gradient. Huang, Sun and Huang (2009) proposed the multi-displacement continuum model for the one-dimensional acoustic metamaterial. Their microstructure continuum model employs additional kinematic variables to represent the mechanical behavior of mass-in-mass system. In similar idea, Zhu and Huang, et al (2011) presented a microstructure continuum model to represent two-dimensional elastic metamaterials and used this continuum model to study wave propagation and band gaps in elastic metamaterial. Recently, Michelitsch and Collet, et al (2014) generalized the nonlocal interparticle interactions in the harmonic approximation for the monoatomic periodic chain. The nonlocal constitutive law was generated 


\section{ACCEPTED MANUSCRIPT}

by constructing a generalized harmonic non-local elastic potential which involves any $m-t h$ differences of displacement field and thus contains complete information of constitutive law. The criteria for "weak" and "strong" nonlocality were provided. Moreover the continuum limit was analyzed rigorously and the scaling behavior of the material constants to guarantee the finiteness of elastic potential was discussed. Michelitsch and Collet (2014) further generalize the constitutive model proposed for the monoatomic chain to the polyatomic periodic linear chain. The dispersive characteristics due to strong nonlocality which cannot be captured by classical lattice model were found and discussed.

In this paper, the continuum model of metamaterial with manmade microstructure (mechanical resonator) is considered. In order to establish an effective continuum model of metamaterial with micro-resonator to keep nearly same dispersive properties with the crystal lattice of metamaterial, the classic continuum model, multi-displacement continuum model and some gradient continuum models are derived from the discrete lattice of metamaterial based on different assumptions. The dispersive curves of these continuum models are compared with each other and with that of discrete lattice of metamaterial. Based on the numerical results, the disadvantages of the classical continuum model and the gradient continuum model for the short wavelength are discussed. It is found that the nonlocal gradient continuum model may be unconditional stable as the gradual decreasing of wavelength provided the nonlocal parameter in the nonlocal gradient continuum model is selected appropriately. 


\section{ACCEPTED MANUSCRIPT}

\section{Dispersive relation and effective mass for the diatomic chain}

Consider a diatomic chain of metamaterial with infinite extension, see Fig.1 (a). The mass of macro-material and micro-material in first atom are indicated by $M_{1}$ and $M_{2}$, respectively, and that in the second atom by $m_{1}$ and $m_{2}$, respectively. The spring coefficient between the atoms of macro-material inside and outside a cell are indicated by $K_{1}^{\prime}$ and $K_{1}$, The spring coefficient between the atoms of macro-material and micro-material in the first atom and that in the second atom are indicated by $K_{2}$ and $K_{2}^{\prime}$, respectively. $u_{1}$ and $u_{2}$ are the displacements of

macro-material and micro-material in each atoms. $u_{s}^{(2(\mathrm{n} \pm j))}$ and $u_{s}^{(2(\mathrm{n} \pm j)+1)}$ are the displacements of the first atom and the second atom, respectively. $L$ is the lattice distance. The motion equation for the macro-material atoms and micro-material atoms in the $n$-th cell are

$$
\begin{array}{r}
M_{1} \frac{d^{2} u_{1}^{(2 n)}}{d t^{2}}=K_{1}^{\prime}\left(u_{1}^{(2 n+1)}-u_{1}^{(2 n)}\right)-K_{1}\left(u_{1}^{(2 n)}-u_{1}^{(2 n-1)}\right)+K_{2}\left(u_{2}^{(2 n)}-u_{1}^{(2 n)}\right) \\
M_{2} \frac{d^{2} u_{2}^{(2 n)}}{d t^{2}}=-K_{2}\left(u_{2}^{(2 n)}-u_{1}^{(2 n)}\right) \\
m_{1} \frac{d^{2} u_{1}^{(2 n+1)}}{d t^{2}}=K_{1}\left(u_{1}^{(2 n+2)}-u_{1}^{(2 n+1)}\right)-K_{1}^{\prime}\left(u_{1}^{(2 n+1)}-u_{1}^{(2 n)}\right)+K_{2}^{\prime}\left(u_{2}^{(2 n+1)}-u_{1}^{(2 n+1)}\right) \\
m_{2} \frac{d^{2} u_{2}^{(2 n+1)}}{d t^{2}}=-K_{2}^{\prime}\left(u_{2}^{(2 n+1)}-u_{1}^{(2 n+1)}\right)
\end{array}
$$

The solutions of the lattice wave are of form

$$
\begin{gathered}
u_{s}^{(2(\mathrm{n} \pm j))}=A_{s} \exp (i(q n L \pm q j L-\omega t)) \\
u_{s}^{(2(\mathrm{n} \pm j)+1)}=B_{s} \exp (i(q n L \pm q j L-\omega t))
\end{gathered}
$$




\section{ACCEPTED MANUSCRIPT}

$A_{s}$ and $B_{s}$ are the amplitude of lattice wave. $s=1$ for the macro-material and $s=2$ for the micro-material in each atom. $q$ is the wave vector and $\omega$ is the angular frequency. $n$ and $j$ are arbitrary integers.

Inserting Eq. (2) into Eq. (1) leads to

$$
\begin{array}{r}
\left(M_{1} \omega^{2}-K_{1}^{\prime}-K_{1}-K_{2}\right) A_{1}+K_{2} A_{2}+\left(K_{1}^{\prime}+K_{1} e^{-i q L}\right) B_{1}=0 \\
K_{2} A_{1}+\left(M_{2} \omega^{2}-K_{2}\right) A_{2}=0 \\
\left(K_{1} e^{1 q L}+K_{1}^{\prime}\right) A_{1}+\left(m_{1} \omega^{2}-K_{1}-K_{1}^{\prime}-K_{2}^{\prime}\right) B_{1}+K_{2}^{\prime} B_{2}=0 \\
K_{2}^{\prime} B_{1}+\left(m_{2} \omega^{2}-K_{2}^{\prime}\right) B_{2}=0
\end{array}
$$

If a non-trivial solution exists, then the coefficient determination needs to be zero, i.e.

$$
\left|\begin{array}{cccc}
M_{1} \omega^{2}-K_{1}^{\prime}-K_{1}-K_{2} & K_{2} & K_{1}^{\prime}+K_{1} e^{-i q L} & 0 \\
K_{2} & M_{2} \omega^{2}-K_{2} & 0 & 0 \\
K_{1} e^{i q L}+K_{1}^{\prime} & 0 & m_{1} \omega^{2}-K_{1}-K_{1}^{\prime}-K_{2}^{\prime} & K_{2}^{\prime} \\
0 & 0 & K_{2}^{\prime} & m_{2} \omega^{2}-K_{2}^{\prime}
\end{array}\right|=0
$$

Eq. (4) is the dispersive equation of lattice wave in the one-dimensional diatomic crystal lattice of metamaterial. Let $\alpha=M_{2} / M_{1}=m_{2} / m_{1}, \quad \beta=K_{2} / K_{1}=K_{2}^{\prime} / K_{1}^{\prime}, \alpha_{0}=M_{1} / m_{1}, \beta_{0}=K_{1}^{\prime} / K_{1}$, $\omega_{0}=\sqrt{K_{1} / M_{1}}$, then, the dispersive equation can be rewritten in a dimensionless form as

$$
\left|\begin{array}{cccc}
\left(\frac{\omega}{\omega_{0}}\right)^{2}-\beta-\beta_{0}-1 & \beta & \beta_{0}+e^{-i(q L)} & 0 \\
\beta & \alpha\left(\frac{\omega}{\omega_{0}}\right)^{2}-\beta & 0 & 0 \\
e^{i(q L)}+\beta_{0} & 0 & \frac{1}{\alpha_{0}}\left(\frac{\omega}{\omega_{0}}\right)^{2}-\beta \beta_{0}-\beta_{0}-1 & \beta \beta_{0} \\
0 & 0 & \beta \beta_{0} & \frac{\alpha}{\alpha_{0}}\left(\frac{\omega}{\omega_{0}}\right)^{2}-\beta \beta_{0}
\end{array}\right|=0
$$




\section{ACCEPTED MANUSCRIPT}

If $K_{2}=0, K_{2}^{\prime}=0, M_{2}=0$ and $m_{2}=0$, then, the diatomic crystal lattice of metamaterial reduces to that of classic material and then the dispersive equation comes down to

$$
\left|\begin{array}{cc}
M \omega^{2}-K_{1}^{\prime}-K_{1} & K_{1}^{\prime}+K_{1} e^{-i q L} \\
K_{1}^{\prime}+K_{1} e^{i q L} & m \omega^{2}-K_{1}^{\prime}-K_{1}
\end{array}\right|=0
$$

Eq. (6) is the dispersive equation of diatomic lattice wave of classic material.

If the diatomic crystal lattice of metamaterial is replaced by that of classic material but the dispersive properties of lattice wave of metamaterial remaining unchanged, then, the dynamical effective masses should be introduced to represent the effects of micro-material. Let the effective masses be indicated by $M_{\text {eff }}$ and $m_{\text {eff }}$. Then, the dispersive equation of the equivalent diatomic chain is

$$
\left|\begin{array}{cc}
M_{e f f} \omega^{2}-K_{1}^{\prime}-K_{1} & K_{1}^{\prime}+K_{1} e^{-i q L} \\
K_{1}^{\prime}+K_{1} e^{i q L} & m_{e f f} \omega^{2}-K_{1}^{\prime}-K_{1}
\end{array}\right|=0
$$

Inserting Eq. (7) into Eq. (4) leads to

$$
m_{e f f}=m_{s t}+\frac{m_{2}\left(\frac{\omega}{\omega_{m 0}}\right)^{2}}{1-\left(\frac{\omega}{\omega_{m 0}}\right)^{2}}, M_{e f f}=M_{s t}+\frac{M_{2}\left(\frac{\omega}{\omega_{M 0}}\right)^{2}}{1-\left(\frac{\omega}{\omega_{M 0}}\right)^{2}}
$$

where $m_{s t}\left(=m_{1}+m_{2}\right)$ and $M_{s t}\left(=M_{1}+M_{2}\right)$ are the static effective masses. $\omega_{m 0}^{2}=\frac{K_{2}^{\prime}}{m_{2}}$ and $\omega_{M 0}^{2}=\frac{K_{2}}{M_{2}}$ are the resonant frequencies of the micro-masses in two atoms.

It is obvious from Eq. (8) that the dynamical effective masses are frequency-dependent. The 


\section{ACCEPTED MANUSCRIPT}

dimensionless effective masses $m_{e f f} / m_{s t}$ and $M_{e f f} / M_{s t}$ as a function of $\omega / \omega_{m 0}$ and $\omega / \omega_{M 0}$ are only determined by the mass ratio $\frac{m_{1}}{m_{2}}$ and $\frac{M_{1}}{M_{2}}$, respectively. Fig.2 shows the frequency-dependent of the dimensionless effective masses. When the frequency tends to the resonant frequency $\omega_{m 0}$ from right side $\left(\omega \geq \omega_{m 0}\right)$, the effective mass $m_{e f f}$ becomes negative. The same argument applies to the effective mass $M_{e f f}$. This is an important feature of metamaterial. Fig. 3 shows the dispersive curves of lattice wave in the diatomic crystal lattice of metamaterial and in the diatomic crystal lattice of classic material. Different from the diatomic lattice wave in the classic material, the diatomic lattice wave in the metamaterial has four branches. Based on the order from bottom to top, the acoustic and the optical branches are similar to that in the classic material but have lower frequency compared with that in the classic material. The third (down concave) and the fourth (upper convex) branches have higher frequency. It is noticed that there are three band gaps for the metamaterial lattice while only one band gap for the classical material lattice. Therefore, there are more large freedoms of design for the metamaterial to modulate the band gap behavior. Fig.4 shows the influences of the mass ratio $\left(M_{2} / M_{1}\right.$ or $\left.m_{2} / m_{1}\right)$ upon the dispersive curves while Fig.5 shows the influences of rigid ratio ( $K_{2} / K_{1}$ or $K_{2}^{\prime} / K_{1}^{\prime}$ ) upon the dispersive curves. It is found that all of the four branches of the dispersive curves shift toward low frequency with the increasing of mass ratio and toward high frequency with the increasing of the rigid ratio. In other word, the mechanical resonator with larger mass and smaller rigid is more likely to create the low frequency band gaps. 


\section{ACCEPTED MANUSCRIPT}

\section{Multi-displacement continuum model}

There is only one degree of freedom and single material constant in the classical continuum model of one-dimensional discrete lattice. On the contrary, there are four masses, namely, $M_{1}$, $M_{2}, m_{1}$ and $m_{2}$, in the metamaterial lattice, as is depicted in Fig.1(a). Obviously, the classical continuum theory is not suitable to represent the continuum model of metamaterial any more. Now we will consider the so-called multi-displacement continuum model which is first proposed in Huang and Sun (2009). First, four dependent displacement fields, which concerned with the apparent mass (macro-material) and the hidden mass (micro-material), are introduced, i.e.

$$
\begin{array}{r}
u_{1}^{(2 n)}(t)=u_{1}(x, t) \\
u_{2}^{(2 n)}(t)=u_{2}(x, t) \\
u_{1}^{(2 n+1)}(t)=u_{1}^{\prime}(x, t) \\
u_{2}^{(2 n+1)}(t)=u_{2}^{\prime}(x, t)
\end{array}
$$

The macro-displacement of the $(2 n+2)$ th atom can be expressed as

$$
u_{1}^{(2 n+2)}=u_{1}(x+L)=u_{1}(x)+\frac{\partial u_{1}}{\partial x} L
$$

Then, the kinetic energy density and potential energy density can be expressed as

$$
\begin{gathered}
W=\frac{1}{2 L}\left[K_{1}\left(u_{1}^{(2 n+2)}-u_{1}^{(2 n+1)}\right)^{2}+K_{1}^{\prime}\left(u_{1}^{(2 n+1)}-u_{1}^{(2 n)}\right)^{2}+\right. \\
\left.K_{2}\left(u_{2}^{(2 n)}-u_{1}^{(2 n)}\right)^{2}+K_{2}^{\prime}\left(u_{2}^{(2 n+1)}-u_{1}^{(2 n+1)}\right)^{2}\right] \\
T=\frac{1}{2 L}\left[M_{1}\left(\dot{u}_{1}^{(2 n)}\right)^{2}+M_{2}\left(\dot{u}_{2}^{(2 n)}\right)^{2}+m_{1}\left(\dot{u}_{1}^{(2 n+1)}\right)^{2}+m_{2}\left(\dot{u}_{2}^{(2 n+1)}\right)^{2}\right]
\end{gathered}
$$




\section{ACCEPTED MANUSCRIPT}

Inserting Eq. (9) and Eq. (10) into Eq. (11) leads to

$$
\begin{array}{r}
W=\frac{1}{2 L}\left[K_{1}\left(u_{1}+\frac{\partial u_{1}}{\partial x} L-u_{1}^{\prime}\right)^{2}+K_{1}^{\prime}\left(u_{1}^{\prime}-u_{1}\right)^{2}+K_{2}\left(u_{2}-u_{1}\right)^{2}+K_{2}^{\prime}\left(u_{2}^{\prime}-u_{1}^{\prime}\right)^{2}\right] \\
T=\frac{1}{2 L}\left[M_{1}\left(\dot{u}_{1}\right)^{2}+M_{2}\left(\dot{u}_{2}\right)^{2}+m_{1}\left(\dot{u}_{1}^{\prime}\right)^{2}+m_{2}\left(\dot{u}_{2}^{\prime}\right)^{2}\right]
\end{array}
$$

We define macro normal strain $\varepsilon=\partial u_{1} / \partial x$, macro relative strain $\varepsilon^{\prime}(x)=\left(u_{1}^{\prime}-u_{1}\right) / L$, micro relative strain $u_{12}(x)=\left(u_{2}-u_{1}\right) / L$ and $u_{22}(x)=\left(u_{2}^{\prime}-u_{1}^{\prime}\right) / L$. Moreover, define the stress and the relative stress

$$
\begin{array}{r}
\sigma=\frac{\partial W}{\partial \varepsilon}=L K_{1} \varepsilon-L K_{1} \varepsilon^{\prime} \\
\sigma^{\prime}=\frac{\partial W}{\partial \varepsilon^{\prime}}=L\left(K_{1}+K_{1}^{\prime}\right) \varepsilon^{\prime}-L K_{1} \varepsilon \\
\sigma_{12}=\frac{\partial W}{\partial u_{12}}=L K_{2} u_{12} \\
\sigma_{22}=\frac{\partial W}{\partial u_{22}}=L K_{2}^{\prime} u_{22}
\end{array}
$$

Eq. (13) represents the constitutive relation of the multi-displacement continuum model. By adopting the Hamilton variation principle,

$$
\delta \int_{t_{0}}^{t_{1}} \int_{V}(T-W) d V d t=0
$$

We obtain the motion equation as

$$
\begin{array}{r}
M_{1} \ddot{u}_{1}-K_{1} L^{2} \frac{\partial^{2} u_{1}}{\partial x^{2}}+\left(K_{1}+K_{1}^{\prime}+K_{2}\right) u_{1}+L K_{1} \frac{\partial u_{1}^{\prime}}{\partial x}-\left(K_{1}+K_{1}^{\prime}\right) u_{1}^{\prime}-K_{2} u_{2}=0 \\
M_{2} \ddot{u}_{2}+K_{2}\left(u_{2}-u_{1}\right)=0 \\
m_{1} \ddot{u}_{1}^{\prime}+\left(K_{1}+K_{1}^{\prime}+K_{2}^{\prime}\right) u_{1}^{\prime}-L K_{1} \frac{\partial u_{1}}{\partial x}-\left(K_{1}+K_{1}^{\prime}\right) u_{1}-K_{2}^{\prime} u_{2}^{\prime}=0 \\
m_{2} \ddot{u}_{2}^{\prime}+K_{2}^{\prime}\left(u_{2}^{\prime}-u_{1}^{\prime}\right)=0
\end{array}
$$




\section{ACCEPTED MANUSCRIPT}

Let the continuous displacement field be of form

$$
\begin{aligned}
& u_{1}=D_{1} \exp (i(q x-\omega t)) \\
& u_{2}=D_{2} \exp (i(q x-\omega t)) \\
& u_{1}^{\prime}=D_{1}^{\prime} \exp (i(q x-\omega t)) \\
& u_{2}^{\prime}=D_{2}^{\prime} \exp (i(q x-\omega t))
\end{aligned}
$$

By inserting them into Eq. (15), the dispersive equation of propagation wave can be obtained in the multi-displacement continuum model.

$$
\left|\begin{array}{cccc}
\left(\frac{\omega}{\omega_{0}}\right)^{2}-(L q)^{2}-\beta-\beta_{0}-1 & \beta & 1+\beta_{0}-(L q) i & 0 \\
\beta & \alpha\left(\frac{\omega}{\omega_{0}}\right)^{2}-\beta & 0 & 0 \\
1+\beta_{0}+(L q) i & 0 & \frac{1}{\alpha_{0}}\left(\frac{\omega}{\omega_{0}}\right)^{2}-\beta \beta_{0}-\beta_{0}-1 & \beta \beta_{0} \\
0 & 0 & \beta \beta_{0} & \frac{\alpha}{\alpha_{0}}\left(\frac{\omega}{\omega_{0}}\right)^{2}-\beta \beta_{0}
\end{array}\right|=0
$$

The dispersive curves predicted by the multi-displacement continuum model are shown in Fig.6.

It is found that the dispersive curves of the first three modes from bottom to top predicted by the multiple displacement continuum model has good agreement with that predicted by the discrete diatomic crystal lattice model at long wavelength range $(q L \leq 1)$. But the dispersive curves of the fourth mode predicted by the multiple displacement continuum model has an agreement with that predicted by the discrete diatomic crystal lattice model only at long wave limit (near $q L=0$ ). The deviation increase gradually as the wavelength decreases gradually. 


\section{ACCEPTED MANUSCRIPT}

\section{Gradient continuum model}

Let Eq. (10) replaced by

$$
\begin{aligned}
& u_{1}^{(2 \mathrm{n}-1)}(x, t)=u_{1}^{\prime}(x-L, t) \\
& =u_{1}^{\prime}-L u_{1, x}^{\prime}+\frac{1}{2 !} L^{2} u_{1, x x}^{\prime}-\frac{1}{3 !} L^{3} u_{1, x x x}^{\prime}+\frac{1}{4 !} L^{4} u_{1, x x x x}^{\prime}-\frac{1}{5 !} L^{5} u_{1, x x x x}^{\prime}+\frac{1}{6 !} L^{6} u_{1, x x x x x}^{\prime}-\ldots \\
& u_{1}^{(2 \mathrm{n}+2)}(x, t)=u_{1}(x+L, t) \\
& =u_{1}+L u_{1, x}+\frac{1}{2 !} L^{2} u_{1, x x}+\frac{1}{3 !} L^{3} u_{1, x x x}+\frac{1}{4 !} L^{4} u_{1, x x x x}+\frac{1}{5 !} L^{5} u_{1, x x x x x}+\frac{1}{6 !} L^{6} u_{1, x x x x x x}+\ldots
\end{aligned}
$$

and Eq.(18) is truncated by six order derivative for calculation purpose. Inserting Eq. (9) and Eq.

(18) into Eq. (1), leads to

$$
\begin{aligned}
& M_{1} \ddot{u}_{1}=K_{1}^{\prime}\left(u_{1}^{\prime}-u_{1}\right)-K_{1}\left(u_{1}-u_{1}^{\prime}+L u_{1, x}^{\prime}-\frac{1}{2 !} L^{2} u_{1, x x}^{\prime}+\frac{1}{3 !} L^{3} u_{1, x x x}^{\prime}-\right. \\
& \left.\frac{1}{4 !} L^{4} u_{1, x x x x}^{\prime}+\frac{1}{5 !} L^{5} u_{1, x x x x}^{\prime}-\frac{1}{6 !} L^{6} u_{1, x x x x x}^{\prime}\right)+K_{2}\left(u_{2}-u_{1}\right) \\
& m_{1} \ddot{u}_{1}^{\prime}=K_{1}\left(u_{1}+L u_{1, x}+\frac{1}{2 !} L^{2} u_{1, x x}+\frac{1}{3 !} L^{3} u_{1, x x x}+\frac{1}{4 !} L^{4} u_{1, x x x x}+\quad(19 \mathrm{c})\right. \\
& \left.\frac{1}{5 !} L^{5} u_{1, x x x x}+\frac{1}{6 !} L^{6} u_{1, x x x x x}-u_{1}^{\prime}\right)-K_{1}^{\prime}\left(u_{1}^{\prime}-u_{1}\right)+K_{2}^{\prime}\left(u_{2}^{\prime}-u_{1}^{\prime}\right) \quad
\end{aligned}
$$

Inserting Eq. (9) and Eq. (18) into Eq. (11), the corresponding potential energy density can be expressed as

$$
W=\frac{1}{2 L}\left[K_{1}\left(\sum_{m=0}^{N} \frac{L^{m}}{m !} \frac{\partial^{m} u_{1}}{\partial x^{m}}-u_{1}^{\prime}\right)^{2}+K_{1}^{\prime}\left(u_{1}^{\prime}-u_{1}\right)^{2}+K_{2}\left(u_{2}-u_{1}\right)^{2}+K_{2}^{\prime}\left(u_{2}^{\prime}-u_{1}^{\prime}\right)^{2}\right]
$$

Here, $N$ is the highest order of the Taylor expansion of the Eqs. (18). The hypothetical strain 


\section{ACCEPTED MANUSCRIPT}

keeps unchanged, and we get

$$
W=\frac{1}{2 L}\left[K_{1} L^{2}\left(\sum_{m=1}^{N} \frac{L^{m-1}}{m !} \frac{\partial^{m-1} \varepsilon}{\partial x^{m-1}}-L \varepsilon^{\prime}\right)^{2}+K_{1}^{\prime} L^{2}\left(\varepsilon^{\prime}\right)^{2}+K_{2} L^{2} u_{12}{ }^{2}+K_{2}^{\prime} L^{2} u_{22}^{2}\right]
$$

The stress and the relative stress can be obtained by

$$
\begin{array}{r}
\sigma=\frac{\partial W}{\partial \varepsilon}=L K_{1} \sum_{m=1}^{N} \frac{L^{m-1}}{m !} \frac{\partial^{m-1} \varepsilon}{\partial x^{m-1}}-L K_{1} \varepsilon^{\prime} \\
\sigma^{\prime}=\frac{\partial W}{\partial \varepsilon^{\prime}}=L\left(K_{1}+K_{1}^{\prime}\right) \varepsilon^{\prime}-L K_{1} \sum_{m=1}^{N} \frac{L^{m-1}}{m !} \frac{\partial^{m-1} \varepsilon}{\partial x^{m-1}} \\
\sigma_{12}=\frac{\partial W}{\partial u_{12}}=L K_{2} u_{12} \\
\sigma_{22}=\frac{\partial W}{\partial u_{22}}=L K_{2}^{\prime} u_{22}
\end{array}
$$

Since $\sigma$ and $\sigma^{\prime}$ are associated with $\varepsilon$ itself and his first to the $(N-1)$ th order derivatives. Eq. (19) is regarded as the fifth fourth order gradient continuum model.

Let

$$
\begin{aligned}
& u_{1}=F_{1} \exp (i(q x-\omega t)) \\
& u_{2}=F_{2} \exp (i(q x-\omega t)) \\
& u_{1}^{\prime}=F_{1}^{\prime} \exp (i(q x-\omega t)) \\
& u_{2}^{\prime}=F_{2}^{\prime} \exp (i(q x-\omega t))
\end{aligned}
$$

Inserting Eq. (23) into Eq. (19) leads to the dispersive equation 


\section{ACCEPTED MANUSCRIPT}

$$
\left|\begin{array}{cccc}
\left(\frac{\omega}{\omega_{0}}\right)^{2}-\beta-\beta_{0}-1 & \beta & \beta_{0}+\gamma_{1}(\mathrm{q}) & 0 \\
\beta & \alpha\left(\frac{\omega}{\omega_{0}}\right)^{2}-\beta & 0 & 0 \\
\gamma_{2}(\mathrm{q})+\beta_{0} & 0 & \frac{1}{\alpha_{0}}\left(\frac{\omega}{\omega_{0}}\right)^{2}-\beta \beta_{0}-\beta_{0}-1 & \beta \beta_{0} \\
0 & 0 & \beta \beta_{0} & \frac{\alpha}{\alpha_{0}}\left(\frac{\omega}{\omega_{0}}\right)^{2}-\beta \beta_{0}
\end{array}\right|=0
$$

where the explicit expressions of $\gamma_{i}$ are given in Appendix A.

Fig.7 shows the dispersive curves of the gradient continuum model. It is found that the dispersive curves corresponding with the gradient continuum model is fitting well with that predicted by the discrete lattice model in wider frequency range compared with the multiple displacement continuum model. The higher the order number of strain gradient considered, the wider the frequency range with satisfying fit. However, the dispersive curves may become unstable when the wavelength decreases gradually. For example, the first and third branches tend to zero when the wavelength goes beyond some certain critical values while the tangent of fourth branch tends to infinite. Though the critical value where the unstable phenomenon begin to appear can be delayed by involving more higher order of the strain gradient in the continuum model, the continuum model with unconditional stability is still expected.

\section{Nonlocal gradient continuum model}

Assume $U^{(n)}(t)$ is the centroid displacement of the $n$-th cell, see Fig.1b. Then, the momentum of single cell can be written as 


\section{ACCEPTED MANUSCRIPT}

$$
m_{e f f}^{\text {cell }} \dot{U}^{(n)}=M_{e f f} \dot{u}_{1}^{(2 n)}+m_{e f f} \dot{u}_{1}^{(2 n+1)}
$$

$m_{\text {eff }}^{\text {cell }}$ is the effective masses of the $n$-th cell and since $u_{1}^{(2 n)}$ is proportional to $u_{1}^{(2 n+1)}$, both $u_{1}^{(2 n)}$ and $u_{1}^{(2 n+1)}$ can be expressed by $U^{(n)}(t)$. Different from the classical continuum hypothesis, $U(x, t)$ is not directly obtained by the continuous field variable $U^{(n)}(t)$, but by

$$
U(x, t)=\frac{1}{1+2 a_{1}}\left\{a_{1} U^{(n-1)}(t)+U^{(n)}(t)+a_{1} U^{(n+1)}(t)\right\}
$$

It implies that the continuous displacement field $U(x, t)$ is the averaged value of the displacements of adjacent several cells. The parameter $a_{1}$ is the weighting coefficient which satisfies $0 \leq a_{1}<1$. The nonlocal effect is taken into account when the continuous displacement field $U(x, t)$ is established by Eq.(26).

In order to obtain the motion equation related to the displacement $U(x, t)$, we should express $U^{(n)}(t), U^{(n \pm 1)}(t)$ in terms of continuous field $U(x, t)$ and its derivatives. We assume that the deviations of $U^{(n)}(t)$ from $U(x, t)$ are small, so that the following relationship may be written:

$$
U^{(\mathrm{n})}(t)=U(x, t)+\sum_{j=1}^{2 N} L^{j} f_{j}(x, t)+O\left(L^{2 N+1}\right)
$$

In order to guarantee the convergence of the series, the following condition is established.

$$
L^{j+1} f_{j+1}(x, t) \ll L^{j} f_{j}(x, t)
$$

These functions of $f_{j}(x, t)$ are unclear so far and should be determined by inserting those discrete displacements into Eq. (26). The displacement of adjacent cells can be determined by the 


\section{ACCEPTED MANUSCRIPT}

application of the Taylor expansions

$$
\begin{aligned}
& U^{(\mathrm{n} \pm 1)}(t)=U(x \pm L, t)+\sum_{j=1}^{2 N} L^{j} f_{j}(x \pm L, t)+O\left(L^{2 N+1}\right) \\
& =\sum_{m=0}^{2 N}( \pm 1)^{m} \frac{L^{m}}{m !} \frac{\partial^{m} U(x, t)}{\partial x^{m}}+\sum_{m=0}^{2 N} \sum_{j=1}^{2 N-m}( \pm 1)^{m} \frac{L^{m+j}}{m !} \frac{\partial^{m} f_{j}(x, t)}{\partial x^{m}}+O\left(L^{2 N+1}\right)
\end{aligned}
$$

Inserting Eqs. (27)-(28) into Eq. (26) leads to

$$
\begin{aligned}
& U(x, t)=\frac{1}{1+2 a_{1}}\left(U(x, t)+\sum_{j=1}^{2 N} L^{j} f_{j}(x, t)\right)+\frac{2 a_{1}}{1+2 a_{1}} \sum_{m=0}^{N} \frac{L^{2 m}}{(2 m) !} \frac{\partial^{2 m} U(x, t)}{\partial x^{2 m}} \\
& +\frac{2 a_{1}}{1+2 a_{1}} \sum_{m=0}^{N} \sum_{j=1}^{2 N-2 m} \frac{L^{2 m+j}}{(2 m) !} \frac{\partial^{2 m} f_{j}(x, t)}{\partial x^{2 m}}+O\left(L^{2 N+1}\right)
\end{aligned}
$$

The operator $L \partial / \partial x$ is supposed to be much smaller than unity, therefore the perturbation method is used with Eq. (29). By comparing the coefficients of $L^{n}$ at both sides of equal mark, the following set of equations are obtained, ordered by the corresponding powers of $L^{n}$

$$
\begin{array}{r}
L^{0}: U=\frac{1}{1+2 a_{1}}\left(U+2 a_{1} U\right) \\
L^{1}: 0=f_{1}+2 a_{1} f_{1} \\
L^{2}: 0=\left(1+2 a_{1}\right) f_{2}+a_{1} \frac{\partial^{2} U}{\partial x^{2}} \\
L^{3}: 0=\left(1+2 a_{1}\right) f_{3}+a_{1} \frac{\partial^{2} f_{1}}{\partial x^{2}} \\
L^{4}: 0=\left(1+2 a_{1}\right) f_{4}+\frac{a_{1}}{12} \frac{\partial^{4} U}{\partial x^{4}}+a_{1} \frac{\partial^{2} f_{2}}{\partial x^{2}} \\
L^{5}: 0=\left(1+2 a_{1}\right) f_{5}+\frac{a_{1}}{12} \frac{\partial^{4} f_{1}}{\partial x^{4}}+a_{1} \frac{\partial^{2} f_{3}}{\partial x^{2}}
\end{array}
$$




\section{ACCEPTED MANUSCRIPT}

$$
L^{6}: 0=\left(1+2 a_{1}\right) f_{6}+\frac{a_{1}}{360} \frac{\partial^{6} U}{\partial x^{6}}+\frac{a_{1}}{12} \frac{\partial^{4} f_{2}}{\partial x^{4}}+a_{1} \frac{\partial^{2} f_{4}}{\partial x^{2}}
$$

which can be generalized as

$$
\begin{gathered}
L^{m}(\mathrm{~m}=1,3,5, \ldots): 0=f_{m}+2 a_{1} \sum_{j=0}^{(\mathrm{m}-1) / 2} \frac{1}{(2 j) !} \frac{\partial^{2 j} f_{m-2 j}(x, t)}{\partial x^{2 j}} \\
L^{m}(\mathrm{~m}=2,4,6, \ldots): 0=f_{m}+2 a_{1} \frac{1}{(m) !} \frac{\partial^{m} U(x, t)}{\partial x^{m}}+2 a_{1} \sum_{j=0}^{m / 2-1} \frac{1}{(2 j) !} \frac{\partial^{2 j} f_{m-2 j}(x, t)}{\partial x^{2 j}}
\end{gathered}
$$

Eq. (30a) is satisfied automatically. By comparing of odd power of $L$, it is found that $f_{j}(j=1,3,5, \ldots)=0$. The same comparison with even power of $L$, we obtain

$$
\begin{array}{r}
f_{2}=-\frac{a_{1}}{1+2 a_{1}} \frac{\partial^{2} U(x, t)}{\partial x^{2}} \\
f_{4}=\frac{a_{1}\left(10 a_{1}-1\right)}{12\left(1+2 a_{1}\right)^{2}} \frac{\partial^{4} U(x, t)}{\partial x^{4}} \\
f_{6}=-\frac{a_{1}\left(1-56 a_{1}+244 a_{1}^{2}\right)}{360\left(1+2 a_{1}\right)^{3}} \frac{\partial^{6} U(x, t)}{\partial x^{6}}
\end{array}
$$

After $f_{j}$ is determined, the expressions of the discrete displacements $U^{(n)}(t)$, and $U^{(n \pm 1)}(t)$ in terms of the continuous displacement field $U(x, t)$ are obtained. It is assumed that the amplitudes of the atoms corresponding to the effective masses $M_{\text {eff }}$ and $m_{\text {eff }}$ in the unit cell be $A$ and $B$, respectively. And the discrete displacement can be derived from Eq. (25).

$$
\begin{gathered}
u_{1}^{(2 n-1)}=\frac{B m_{e f f}^{\text {cell }}}{\left(M_{e f f} A+m_{e f f} B\right)} U^{(n-1)}(t) \\
u_{1}^{(2 n)}=\frac{A m_{e f f}^{\text {cell }}}{\left(M_{e f f} A+m_{e f f} B\right)} U^{(n)}(t)
\end{gathered}
$$




\section{ACCEPTED MANUSCRIPT}

$$
\begin{gathered}
u_{1}^{(2 n+1)}=\frac{B m_{\text {eff }}^{\text {cell }}}{\left(M_{\text {eff }} A+m_{\text {eff }} B\right)} U^{(n)}(t) \\
u_{1}^{(2 n+2)}=\frac{A m_{\text {eff }}^{\text {cell }}}{\left(M_{\text {eff }} A+m_{\text {eff }} B\right)} U^{(n+1)}(t)
\end{gathered}
$$

In order to get the motion equation of $U(x, t)$, Inserting Eq. (33) into the motion equation of discrete diatomic lattice of metamaterial, i.e. Eq. (1), we obtain

$$
\begin{gathered}
M_{e f f} \frac{\partial^{2} U^{(n)}(t)}{\partial t^{2}}=\frac{K_{1}^{\prime} B-K_{1}^{\prime} A-K_{1} A}{A} U^{(n)}(t)+\frac{K_{1} B}{A} U^{(n-1)}(t) \\
m_{e f f} \frac{\partial^{2} U^{(n)}(t)}{\partial t^{2}}=\frac{-K_{1} B-K_{1}^{\prime} B+K_{1}^{\prime} A}{B} U^{(n)}(t)+\frac{K_{1} A}{B} U^{(n+1)}(t)
\end{gathered}
$$

Inserting Eqs. (27)-(28) into Eq. (34) leads to

$$
\begin{aligned}
& M_{e f f}\left(\frac{\partial^{2} U(x, t)}{\partial t^{2}}+\sum_{j=1}^{2 N} L^{j} \frac{\partial^{2} f_{j}(x, t)}{\partial t^{2}}\right)=\frac{K_{1}^{\prime} B-K_{1}^{\prime} A-K_{1} A}{A}\left(U(x, t)+\sum_{j=1}^{2 N} L^{j} f_{j}(x, t)\right)+ \\
& \frac{K_{1} B}{A}\left(\sum_{m=0}^{2 N}(-1)^{m} \frac{L^{m}}{m !} \frac{\partial^{m} U(x, t)}{\partial x^{m}}+\sum_{m=0}^{2 N} \sum_{j=1}^{2 N-m}(-1)^{m} \frac{L^{m+j}}{m !} \frac{\partial^{m} f_{j}(x, t)}{\partial x^{m}}\right)+O\left(L^{2 N+1}\right) \\
& m_{e f f}\left(\frac{\partial^{2} U(x, t)}{\partial t^{2}}+\sum_{j=1}^{2 N} L^{j} \frac{\partial^{2} f_{j}(x, t)}{\partial t^{2}}\right)=\frac{-K_{1} B-K_{1}^{\prime} B+K_{1}^{\prime} A}{B}\left(U(x, t)+\sum_{j=1}^{2 N} L^{j} f_{j}(x, t)\right)+ \\
& \frac{K_{1} A}{B}\left(\sum_{m=0}^{2 N} \frac{L^{m}}{m !} \frac{\partial^{m} U(x, t)}{\partial x^{m}}+\sum_{m=0}^{2 N} \sum_{j=1}^{2 N-m} \frac{L^{m+j}}{m !} \frac{\partial^{m} f_{j}(x, t)}{\partial x^{m}}\right)+O\left(L^{2 N+1}\right)
\end{aligned}
$$

Truncating the infinite series in Eq. (35) by $N=3$, we obtain

$$
\begin{aligned}
& m_{e f f}\left(\frac{\partial^{2} U}{\partial t^{2}}-\lambda_{1} \frac{\partial^{2} U}{\partial x^{2}}+\beta_{1} \frac{\partial U}{\partial x}\right)-\frac{a_{1} L^{2} M_{e f f}}{1+2 a_{1}} \frac{\partial^{2} U}{\partial x^{2}}\left(\frac{\partial^{2} U}{\partial t^{2}}-\lambda_{2} \frac{\partial^{2} U}{\partial x^{2}}+\beta_{2} \frac{\partial U}{\partial x}\right)+ \\
& \frac{a_{1}\left(10 a_{1}-1\right) L^{4} M_{e f f}}{12\left(1+2 a_{1}\right)^{2}} \frac{\partial^{4} U}{\partial x^{4}}\left(\frac{\partial^{2} U}{\partial t^{2}}-\lambda_{3} \frac{\partial^{2} U}{\partial x^{2}}+\beta_{3} \frac{\partial U}{\partial x}\right)+\lambda U=0
\end{aligned}
$$




\section{ACCEPTED MANUSCRIPT}

$$
\begin{aligned}
& m_{e f f}\left(\frac{\partial^{2} U}{\partial t^{2}}-\lambda_{1}^{\prime} \frac{\partial^{2} U}{\partial x^{2}}+\beta_{1}^{\prime} \frac{\partial U}{\partial x}\right)-\frac{a_{1} L^{2} M_{e f f}}{1+2 a_{1}} \frac{\partial^{2} U}{\partial x^{2}}\left(\frac{\partial^{2} U}{\partial t^{2}}-\lambda_{2}^{\prime} \frac{\partial^{2} U}{\partial x^{2}}+\beta_{2}^{\prime} \frac{\partial U}{\partial x}\right)+ \\
& \frac{a_{1}\left(10 a_{1}-1\right) L^{4} M_{e f f}}{12\left(1+2 a_{1}\right)^{2}} \frac{\partial^{4} U}{\partial x^{4}}\left(\frac{\partial^{2} U}{\partial t^{2}}-\lambda_{3}^{\prime} \frac{\partial^{2} U}{\partial x^{2}}+\beta_{3}^{\prime} \frac{\partial U}{\partial x}\right)+\lambda^{\prime} U=0
\end{aligned}
$$

Similarly, truncating the infinite series by $N=2$, we obtain

$$
\begin{aligned}
& M_{e f f}\left(\frac{\partial^{2} U}{\partial t^{2}}-\lambda_{1} \frac{\partial^{2} U}{\partial x^{2}}+\beta_{1} \frac{\partial U}{\partial x}\right)-\frac{a_{1} L^{2} M_{e f f}}{1+2 a_{1}} \frac{\partial^{2} U}{\partial x^{2}}\left(\frac{\partial^{2} U}{\partial t^{2}}-\lambda_{2} \frac{\partial^{2} U}{\partial x^{2}}+\beta_{2} \frac{\partial U}{\partial x}\right)+\lambda U=0 \\
& m_{e f f}\left(\frac{\partial^{2} U}{\partial t^{2}}-\lambda_{1}^{\prime} \frac{\partial^{2} U}{\partial x^{2}}+\beta_{1}^{\prime} \frac{\partial U}{\partial x}\right)-\frac{a_{1} L^{2} M_{e f f}}{1+2 a_{1}} \frac{\partial^{2} U}{\partial x^{2}}\left(\frac{\partial^{2} U}{\partial t^{2}}-\lambda_{2}^{\prime} \frac{\partial^{2} U}{\partial x^{2}}+\beta_{2}^{\prime} \frac{\partial U}{\partial x}\right)+\lambda^{\prime} U=0
\end{aligned}
$$

The potential energy density can be expressed as

$$
W=\frac{1}{2 L}\left[K_{1}\left(U^{(n+1)}-U^{(n)}\right)\right]^{2}
$$

Inserting Eqs. (27)-(28) and Eqs. (32) into Eq. (38) leads to

$$
W=\frac{K_{1}}{2 L}\left(\sum_{m=1}^{2 N} \frac{L^{m}}{m !} \frac{\partial^{m} U(x, t)}{\partial x^{m}}+\sum_{m=1}^{2 N} \sum_{j=1}^{N-[m / 2]} \xi_{2 j} \frac{L^{m+2 j}}{m !} \frac{\partial^{m+2 j} U(x, t)}{\partial x^{m+2 j}}\right)^{2}
$$

Where $[\mathrm{m} / 2]$ indicates the largest integer less than or equal to $m / 2$. The existence of the nonlocal parameter $a_{1}$ can balance the potential energy density to obtain a suitable model matching with the lattice model. Here $j \in N^{+}$, we define normal strain $\varepsilon=\partial U / \partial x . \xi_{2 j}$ is the

corresponding coefficient when Eq. (32) is rewritten in the form of $f_{2 j}=\xi_{2 j} \partial^{2 j} U / \partial x^{2 j}$. Then, the stress is obtained by

$$
\sigma=\frac{\partial W}{\partial \varepsilon}=K_{1} L\left(\sum_{m=1}^{2 N} \frac{L^{m-1}}{m !} \frac{\partial^{m-1} \varepsilon}{\partial x^{m-1}}+\sum_{m=1}^{2 N} \sum_{j=1}^{N-[m / 2]} \xi_{2 j} \frac{L^{m+2 j-1}}{m !} \frac{\partial^{m+2 j-1} \varepsilon}{\partial x^{m+2 j-1}}\right)
$$

Since $\sigma$ is associated with $\varepsilon$ itself and his first to the $(2 N-1) t h$ order derivatives. Eq. (36) and Eq. (37) are regarded as the fifth and the third order nonlocal gradient continuum 


\section{ACCEPTED MANUSCRIPT}

models.

Let the solution be of form

$$
U=C \exp (i(q x-\omega t))
$$

Inserting Eq. (41) into Eq. (36) leads to

$$
\begin{aligned}
& \left(-g_{1}\left(\left(\frac{\omega}{\omega_{0}}\right)^{2}\right)+\lambda_{1} \cdot(q L)^{2}+\beta_{1} \cdot(q L) i\right)+\frac{a_{1}}{1+2 a_{1}}(q L)^{2}\left(-g_{1}\left(\left(\frac{\omega}{\omega_{0}}\right)^{2}\right)+\lambda_{2} \cdot(q L)^{2}+\beta_{2} \cdot(q L) i\right)- \\
& \frac{a_{1}\left(10 a_{1}-1\right)}{12\left(1+2 a_{1}\right)^{2}}(q L)^{4}\left(-g_{1}\left(\left(\frac{\omega}{\omega_{0}}\right)^{2}\right)+\lambda_{3} \cdot(q L)^{2}+\beta_{3} \cdot(q L) i\right)+\lambda=0 \\
& \left(-g_{2}\left(\left(\frac{\omega}{\omega_{0}}\right)^{2}\right)+\lambda_{1}^{\prime} \cdot(q L)^{2}+\beta_{1}^{\prime} \cdot(q L) i\right)+\frac{a_{1}}{1+2 a_{1}}(q L)^{2}\left(-g_{2}\left(\left(\frac{\omega}{\omega_{0}}\right)^{2}\right)+\lambda_{2}^{\prime} \cdot(q L)^{2}+\beta_{2}^{\prime} \cdot(q L) i\right)- \\
& \frac{a_{1}\left(10 a_{1}-1\right)}{12\left(1+2 a_{1}\right)^{2}}(q L)^{4}\left(-g_{2}\left(\left(\frac{\omega}{\omega_{0}}\right)^{2}\right)+\lambda_{3}^{\prime} \cdot(q L)^{2}+\beta_{3}^{\prime} \cdot(q L) i\right)+\lambda^{\prime}=0
\end{aligned}
$$

Similarly, inserting Eq. (41) into Eq. (37) leads to

$$
\begin{aligned}
& \left(-g_{1}\left(\left(\frac{\omega}{\omega_{0}}\right)^{2}\right)+\lambda_{1} \cdot(q L)^{2}+\beta_{1} \cdot(q L) i\right)+\frac{a_{1}}{1+2 a_{1}}(q L)^{2}\left(-g_{1}\left(\left(\frac{\omega}{\omega_{0}}\right)^{2}\right)+\lambda_{2} \cdot(q L)^{2}+\beta_{2} \cdot(q L) i\right)+\lambda=0 \\
& \left(-g_{2}\left(\left(\frac{\omega}{\omega_{0}}\right)^{2}\right)+\lambda_{1}^{\prime}(q L)^{2}+\beta_{1}^{\prime}(q L) i\right)+\frac{a_{1}}{1+2 a_{1}}(q L)^{2}\left(-g_{2}\left(\left(\frac{\omega}{\omega_{0}}\right)^{2}\right)+\lambda_{2}^{\prime}(q L)^{2}+\beta_{2}^{\prime}(q L) i\right)+\lambda^{\prime}=0 \\
& \lambda^{\prime}=0
\end{aligned}
$$

Where the explicit expressions of $g_{i}, \lambda_{i}, \beta_{i}, \lambda, \lambda_{i}^{\prime}, \beta_{i}^{\prime}, \lambda^{\prime}$ are given in Appendix B.

Fig.8 shows the dispersive curves of the nonlocal gradient continuum model. It is found that 


\section{ACCEPTED MANUSCRIPT}

the dispersive curves predicted by the nonlocal gradient continuum model can fit that predicted by the discrete lattice model in wider frequency range compared with the multiple displacement continuum model, meanwhile, the nonlocal gradient continuum model can give unconditional stable dispersive curves compared with the gradient continuum model. Although the nonlocal gradient continuum model is the most potential model among the above three kinds of continuous models, the actual predict effects are dependent upon the choice of the nonlocal parameter $a_{1}$.

Fig.9 and Fig.10 show the effects of nonlocal parameter $a_{1}$ on the dispersive curves. It is found that the increase of $a_{1}$ can help to get stable dispersive curves. If the nonlocal parameter $a_{1}$ fall in $0 \leq a_{1}<0.26$ in the third gradient and $0 \leq a_{1}<0.27$ in the fifth gradient, the acoustic mode and the third mode attenuate in advance. In particular, when $a_{1}=0$, the higher-order dynamical terms, $s_{1} \partial^{4} u_{1} / \partial x^{2} \partial t^{2}$ in the third nonlocal gradient model, as well as $s_{1} \partial^{4} u_{1} / \partial x^{2} \partial t^{2}$ and $s_{2} \partial^{6} u_{1} / \partial x^{4} \partial t^{2}$ in the fifth nonlocal gradient model vanish and the corresponding nonlocal gradient models reduce to the local gradient model in section 4 . On the other hand, if the nonlocal parameter $a_{1}$ satisfies $a_{1} \geq 0.26$ in the third nonlocal gradient model and $a_{1} \geq 0.27$ in the fifth nonlocal gradient model, the dispersive curves are always stable, namely, the dispersive curve does not attenuate in advance and trends to its asymptote.

\section{Conclusions}

Compared with the classical material, the metamaterial has distributed mechanical local resonators. The existence of local resonators results in unique nature of the acoustic metamaterial. 


\section{ACCEPTED MANUSCRIPT}

The appropriate discrete representation of metamaterial is the mass-in-mass plus spring system. The dispersive curves of lattice wave in crystal lattice of metamaterial have more band gaps compared with the classical material. In particular, the low frequency bandgap between the acoustic mode and the optical mode is easier to appear. Some continuum models of the acoustic metamaterial are studied in the present work and some conclusions can be drawn as following.

1) The classical continuum model of discrete lattice cannot predict the bandgap nature because only a single acoustic branch is predicted. In order to predict the band gap nature of metamaterial, the classic continuum model results in the frequency-dependent negative effective mass and the infinite mass at the certain frequency which is physically unacceptable.

2) The multiple displacement continuum model introduces additional degree of freedom of micro-motion and thus predicts not only the low frequency band gap but also avoid the introduction of negative mass and infinite mass. The acoustic branch predicted by the multiple displacement continuum model has good agreement with that of lattice mode. However, the optical branch has good agreement with that of lattice model only for elastic wave with long wavelength $(q L<1)$. The deviation increases evidently as the wavelength decreases.

3) The gradient continuum model can evidently improve the prediction decision and the applied frequency range of the multiple displacement continuum model. But the dispersive curves predicted by the gradient continuum model are not unconditionally stable when the wavelength becomes short gradually. 


\section{ACCEPTED MANUSCRIPT}

4) The nonlocal strain gradient continuum model takes the nonlocal effects into consideration when establishing the continuous displacement field from the discrete displacement of adjacent atoms. By appropriate selection of the nonlocal parameter, the nonlocal gradient continuum model is unconditional stable and can predict physically realistic dispersive curves at total frequency range.

\section{Funding}

The work is supported by the National Natural Science Foundation of China (No.10972029) and Opening fund of State Key Laboratory of Nonlinear Mechanics (LNM) 


\section{ACCEPTED MANUSCRIPT}

\section{References}

1. Askes H, Aifantis E C, 2011. Gradient elasticity in statics and dynamics: An overview of formulations, length scale identification procedures, finite element implementations and new results, Int. J. Solids Struct., 48: 1962-1990.

2. Fang N, Xi D, Xu J, et al, 2006. Ultrosonic metamaterial with negative modulus, Natur. Mater., 5: 452-456.

3. Huang H, Sun C, Huang G, 2009. On the negative effective mass density in acoustic metamaterials, Int. J. Eng. Sci., 47: 610-617.

4. Huang H, Sun C, 2009. Wave attenuation mechanism in an acousticmetamaterial with negative effective mass density, New. J. Phys., 11: 013003.

5. Huang G, Sun C, 2010. Band gaps in a multiresonator acoustic metamaterial, J. Vib. Acoust., 132: 031003.

6. Kadic M, Bückmann T, Stenger N, Thiel M, Wegener M, 2012. On the practicability of pentamode mechanical metamaterials, Appl. Phys. Lett., 100: 191901.

7. Liu Z Y, Zhang X X, Mao Y W, et al, 2000. Locally resonant sonic materials, Sci., 289: 1734-1736.

8. Li J, Chan C T, Double-negative acoustic metamaterials, Phys. Rev. E, 2004, 70: 055602.

9. Lu M H, Fang L, Chen Y F, 2009. Phononic crystals and acoustic metamaterials, Mater. today, 12: 34-42. 


\section{ACCEPTED MANUSCRIPT}

10. Lee S H, Park C M, 2009. Acoustic metamaterial with negative density, Phys. Lett. A, 373: 4464-4469.

11. Mindlin R D, 1964. Micro-structure in linear elasticity, Arch. Ration. Mech. Anal., 16: $51-78$.

12. Metrikine A V, Askes H, 2002. One-dimensional dynamically consistent gradient elasticity models derived from a discrete microstructure, Eur. J. Mech. A-Solid, 21: 555-572.

13. Milton G W, Willis J R, 2007. On modifications of Newton's second law and linear continuum elastodynamics, Proc. R. Soc. A, 463: 855-880.

14. Michelitsch T M, Collet B, Wang X, 2014. Nonlocal constitutive laws generated by matrix functions: Lattice dynamics models and their continuum limits, Int. J. Engng. Sci, 80: $106-123$.

15. Michelitsch T M, Collet B, 2014. A nonlocal constitutive model generated by matrix functions for polyatomic periodic linear chains, Arch. Appl. Mech., 84: 1477-1500.

16. Pendry J B, 2000. Negative refraction makes a perfect lens, Phys. Rev. Lett., 85: 3966-39669.

17. Sheng P, Mei J, Liu Z Y, Wen W J, 2007. Dynamic mass density and acoustic metamaterials, Physica B, 394: 256-261.

18. Zhu R, Huang H, Huang G L, et al, 2011. Microstructure continuum modeling of an elastic metamaterial, Int. J. Eng. Sci., 49: 1477-1485. 


\section{ACCEPTED MANUSCRIPT}

\section{Appendix A}

$\gamma_{1}(\mathrm{q})=1-L q i-\frac{1}{2 !} L^{2} q^{2}+\frac{1}{3 !} L^{3} q^{3} i+\frac{1}{4 !} L^{4} q^{4}-\frac{1}{5 !} L^{5} q^{5} i-\frac{1}{6 !} L^{6} q^{6}$,

$\gamma_{2}(q)=1+L q i-\frac{1}{2 !} L^{2} q^{2}-\frac{1}{3 !} L^{3} q^{3} i+\frac{1}{4 !} L^{4} q^{4}+\frac{1}{5 !} L^{5} q^{4} i-\frac{1}{6 !} L^{6} q^{6}$.

\section{Appendix B}

$$
\begin{aligned}
& g_{1}\left(\left(\frac{\omega}{\omega_{0}}\right)^{2}\right)=(1+\alpha)\left(\frac{\omega}{\omega_{0}}\right)^{2}+\frac{\alpha\left(\frac{\omega}{\omega_{0}}\right)^{2}}{\frac{\beta}{\alpha}-\left(\frac{\omega}{\omega_{0}}\right)^{2}}, \\
& g_{2}\left(\left(\frac{\omega}{\omega_{0}}\right)^{2}\right)=\frac{K_{1}(1+\alpha)}{\alpha_{0}}\left(\frac{\omega}{\omega_{0}}\right)^{2}+\frac{\frac{\alpha K_{1}}{\alpha_{0}}\left(\frac{\omega}{\omega_{0}}\right)^{2}}{\frac{\alpha_{0} \beta \beta_{0}}{\alpha}-\left(\frac{\omega}{\omega_{0}}\right)^{2}}, \\
& \lambda=\frac{\left(1+\beta_{0}\right)(A-B)}{A}, \lambda^{\prime}=\frac{\left(1+\beta_{0}\right)(B-A)}{B}, \lambda_{1}=-\frac{a_{1}\left(1+\beta_{0}\right)(B-A)}{\left(1+2 a_{1}\right) A}+\frac{B}{2 A}, \\
& \lambda_{1}^{\prime}=-\frac{a_{1}\left(1+\beta_{0}\right)(A-B)}{\left(1+2 a_{1}\right) B}+\frac{A}{2 B}, \beta_{1}=\frac{B}{A}, \beta_{1}^{\prime}=-\frac{A}{B}, \\
& \lambda_{2}=-\frac{\left(10 a_{1}-1\right)\left(1+\beta_{0}\right)(B-A)}{12\left(1+2 a_{1}\right) A}+\frac{B}{2 A}-\frac{\left(1+2 a_{1}\right) B}{24 a_{1} A}, \\
& \lambda_{2}^{\prime}=-\frac{\left(10 a_{1}-1\right)\left(1+\beta_{0}\right)(A-B)}{12\left(1+2 a_{1}\right) B}+\frac{A}{2 B}-\frac{\left(1+2 a_{1}\right) A}{24 a_{1} B}, \\
& \beta_{2}=-\frac{\left(1+2 a_{1}\right) B}{6 a_{1} A}+\frac{B}{A}, \beta_{2}^{\prime}=\frac{\left(1+2 a_{1}\right) A}{6 a_{1} B}-\frac{A}{B}, \\
& \lambda_{3}=-\frac{\left(1-56 a_{1}+244 a_{1}^{2}\right)\left(1+\beta_{0}\right)(B-A)}{30\left(1+2 a_{1}\right)\left(10 a_{1}-1\right) A}+\frac{B}{2 A M_{e f f}}-\frac{\left(1+2 a_{1}\right)^{2} B}{2\left(10 a_{1}-1\right) A}+\frac{\left(1+2 a_{1}\right)^{2} B}{60 a_{1}\left(10 a_{1}-1\right) A},
\end{aligned}
$$




\section{ACCEPTED MANUSCRIPT}

$$
\begin{aligned}
& \lambda_{3}^{\prime}=-\frac{\left(1-56 a_{1}+244 a_{1}^{2}\right)\left(1+\beta_{0}\right)(A-B)}{30\left(1+2 a_{1}\right)\left(10 a_{1}-1\right) B}+\frac{A}{2 B}-\frac{\left(1+2 a_{1}\right)^{2} A}{2\left(10 a_{1}-1\right) B}+\frac{\left(1+2 a_{1}\right)^{2} A}{60 a_{1}\left(10 a_{1}-1\right) B}, \\
& \beta_{3}=\frac{\left(1+2 a_{1}\right)^{2} B}{10 a_{1}\left(10 a_{1}-1\right) A}+\frac{B}{A}-\frac{2\left(1+2 a_{1}\right) B}{\left(10 a_{1}-1\right) A}, \beta_{3}^{\prime}=-\frac{\left(1+2 a_{1}\right)^{2} A}{10 a_{1}\left(10 a_{1}-1\right) \mathrm{B}}-\frac{A}{B}+\frac{2\left(1+2 a_{1}\right) A}{\left(10 a_{1}-1\right) \mathrm{B}} .
\end{aligned}
$$




\section{ACCEPTED MANUSCRIPT}

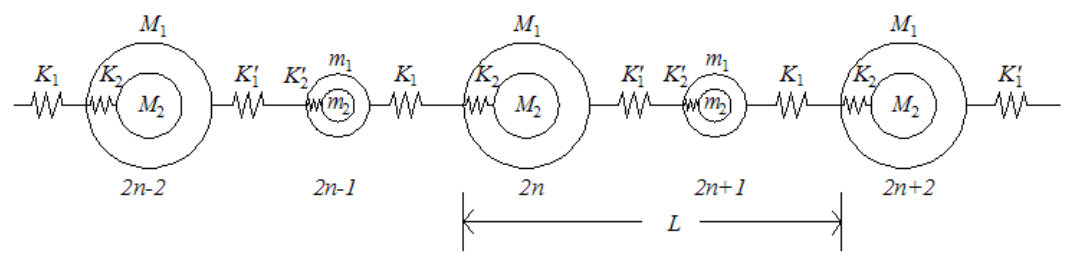

(a)

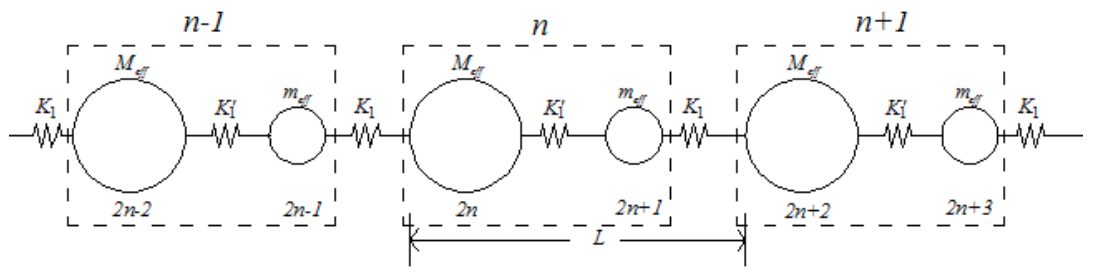

(b)

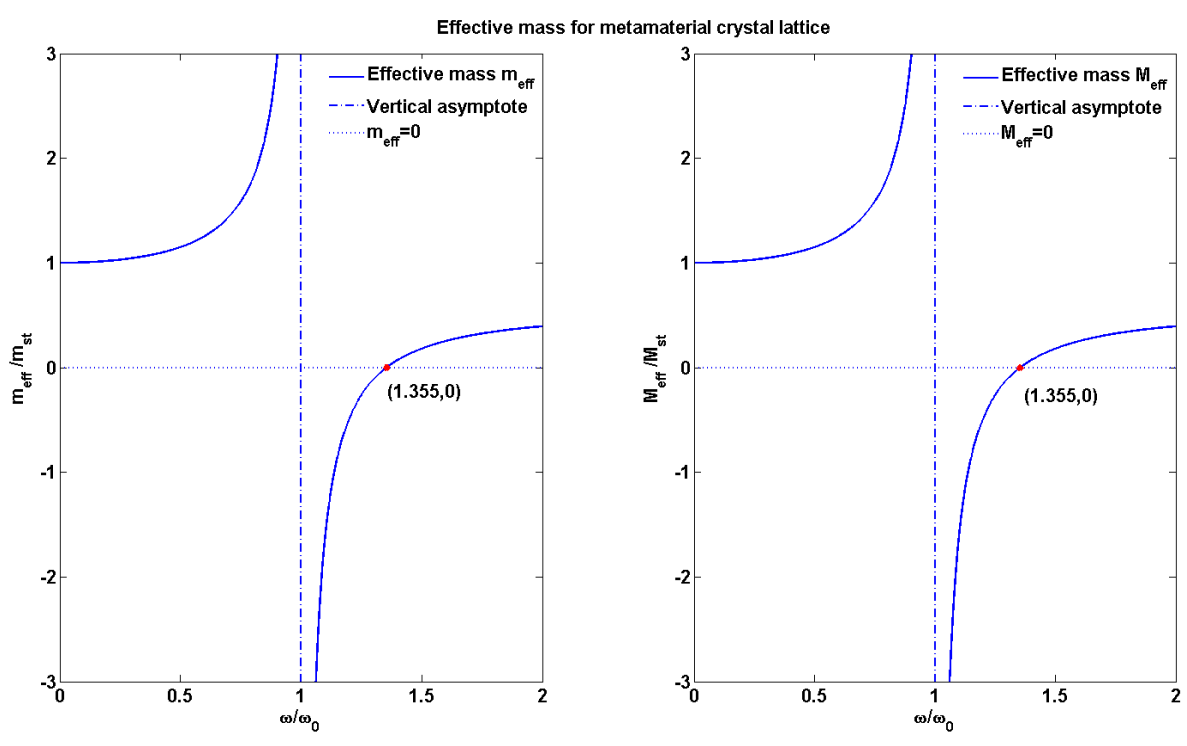

Fig.2 Dimensionless effective masses $m_{e f f} / m_{s t}$ and $M_{\text {eff }} / M_{s t}$ as a function of $\omega / \omega_{0}$. 


\section{ACCEPTED MANUSCRIPT}

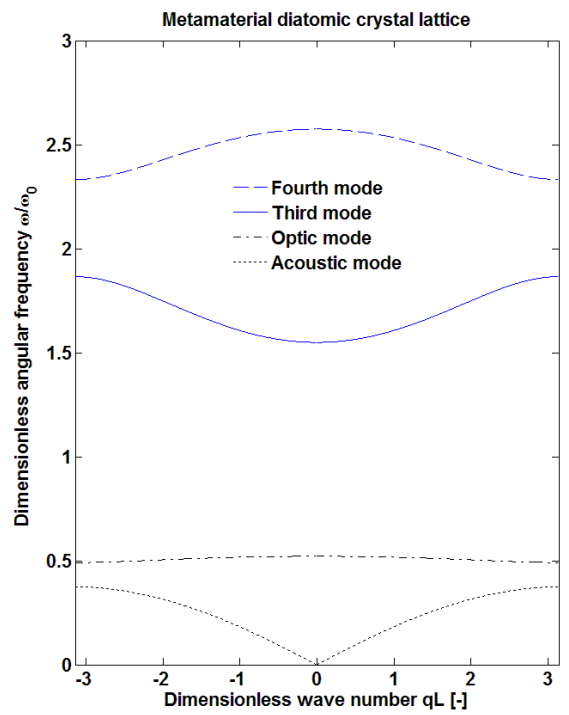

(a)

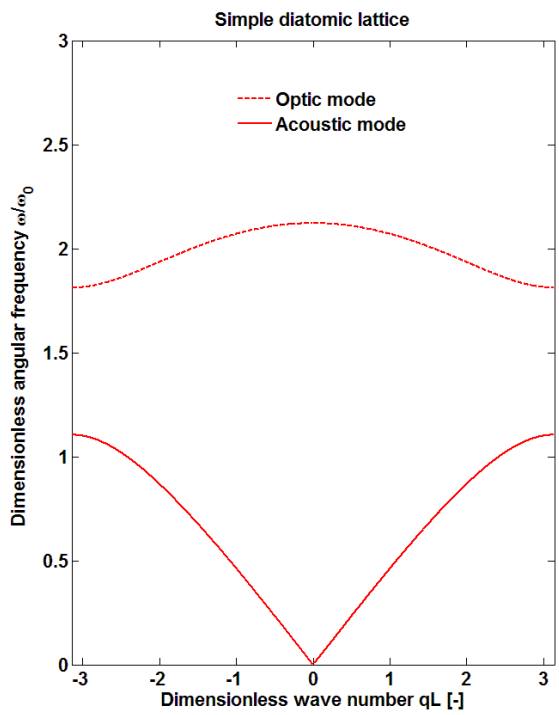

(b)

Fig.3 The dimensionless dispersion curves. (a) metamaterial diatomic crystal lattice with mass in mass; (b) simple diatomic lattice with mass $\left(m=m_{1}, \quad M=M_{1}\right)$. 


\section{ACCEPTED MANUSCRIPT}
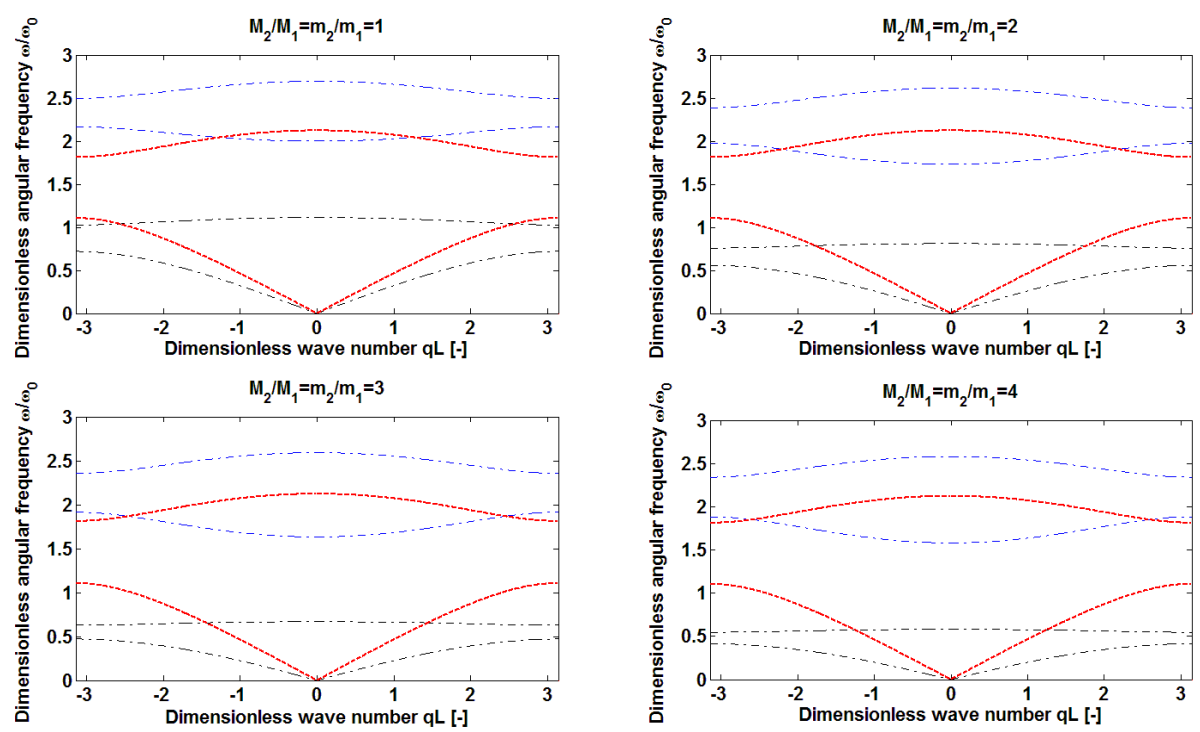

Fig.4 The dimensionless dispersion curves (dot dash line) for different parameter ratio $M_{2} / M_{1}$ or $m_{2} / m_{1}$ compared with that (dashed line) of the simple diatomic lattice

$$
\left(M_{2} / M_{1}=m_{2} / m_{1}, K_{2} / K_{1}=K_{2}^{\prime} / K_{1}^{\prime}=2, M_{1} / m_{1}=2, K_{1}^{\prime} / K_{1}=0.5, \omega_{0}^{2}=K_{1} / m_{1}\right) .
$$




\section{ACCEPTED MANUSCRIPT}
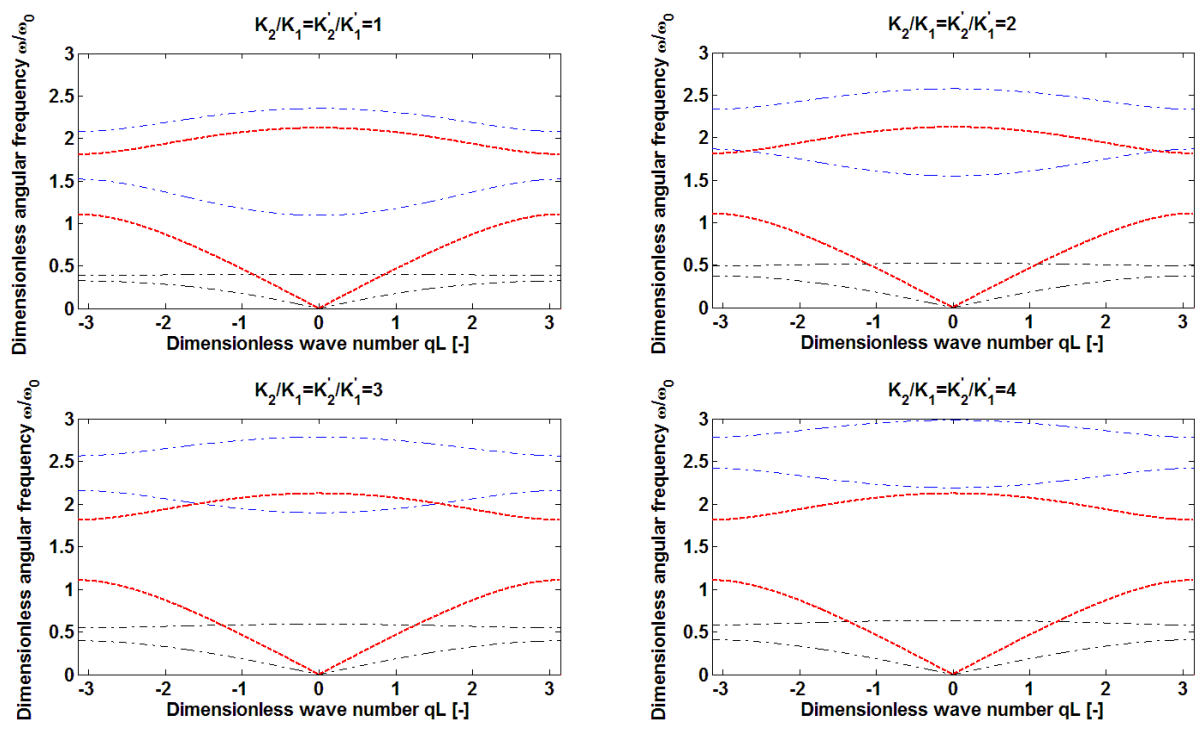

Fig.5 The dimensionless dispersion curves (dot dash line) for different parameter ratio $K_{2} / K_{1}$ or $K_{2}^{\prime} / K_{1}^{\prime}$ compared with that (dotted line) of the simple diatomic lattice $\left(M_{2} / M_{1}=m_{2} / m_{1}=5, K_{2} / K_{1}=K_{2}^{\prime} / K_{1}^{\prime}\right.$,

$$
\left.M_{1} / m_{1}=2, K_{1}^{\prime} / K_{1}=0.5, \omega_{0}^{2}=K_{1} / m_{1}\right)
$$




\section{ACCEPTED MANUSCRIPT}

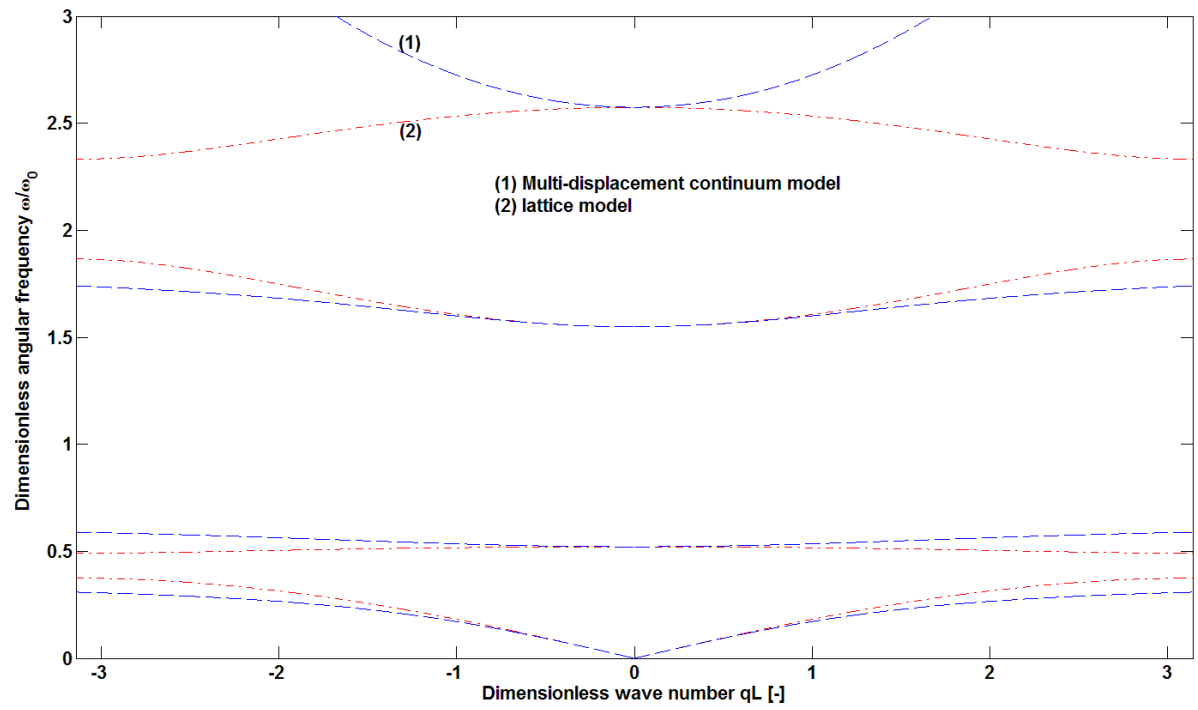

Fig.6 The dimensionless dispersion curves for the metamaterial crystal lattice model (dot dash line) and the multi-displacement continuum model (dotted line). 


\section{ACCEPTED MANUSCRIPT}

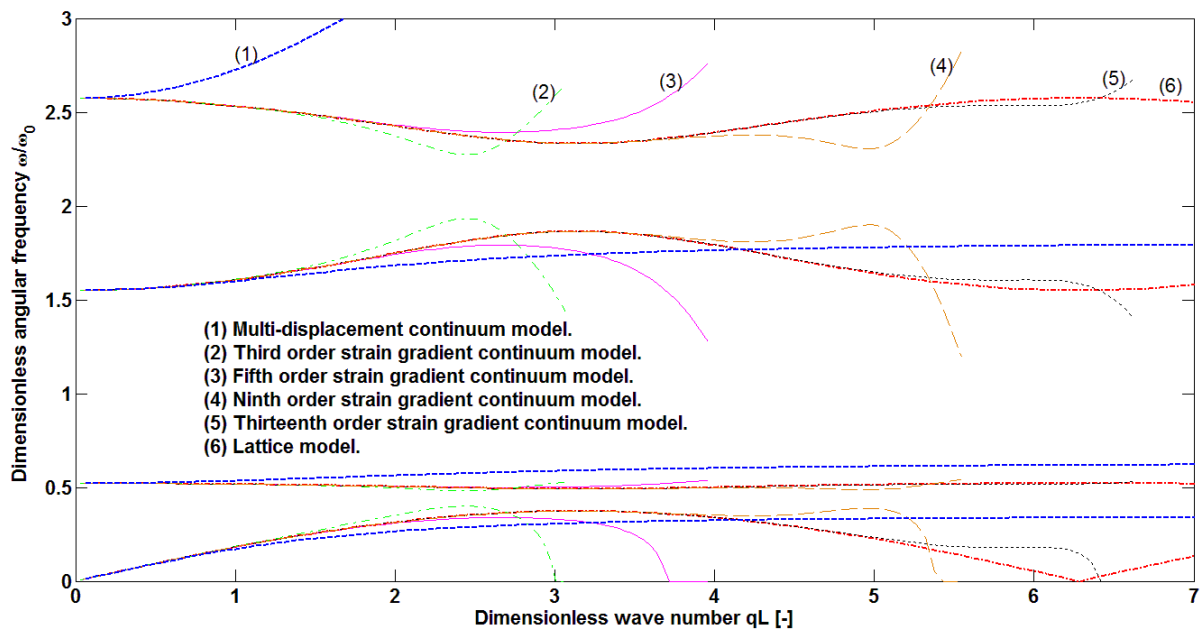

Fig.7 The dimensionless dispersion curves for some order of strain gradient continuum models compared with the lattice model and the multi-displacement continuum model. 


\section{ACCEPTED MANUSCRIPT}

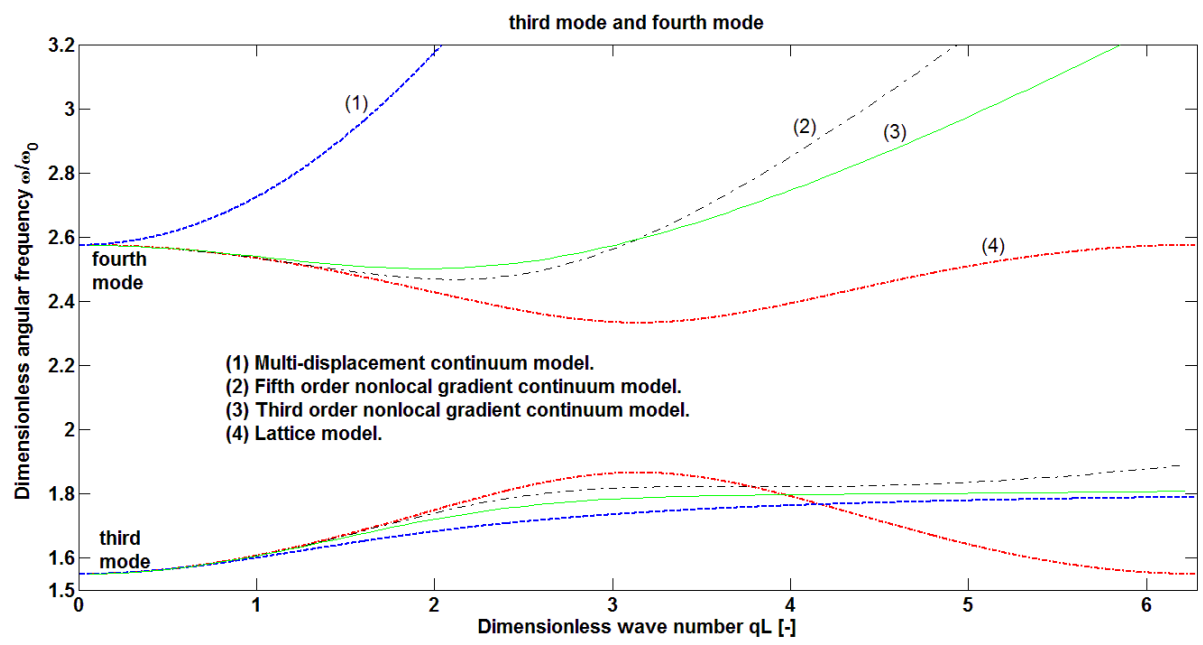

(a)

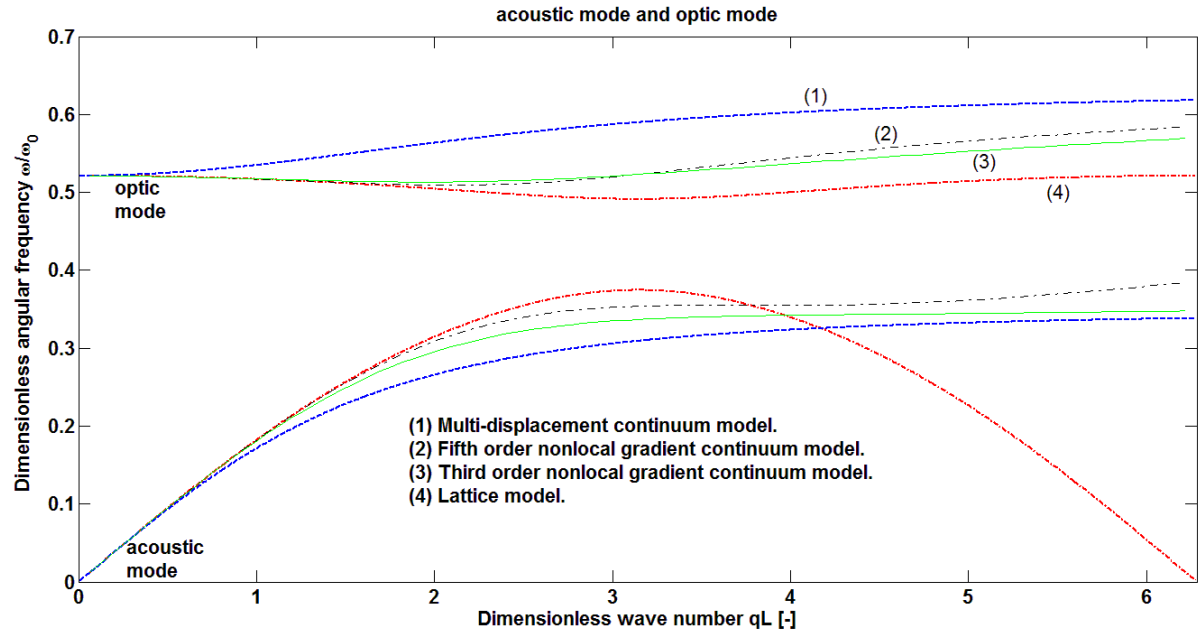

(b)

Fig.8 The dimensionless dispersion curves with $a_{1}=0.3$ for the third order secend and the fifth order fourth nonlocal gradient continuum models compared with the lattice model and the multi-displacement continuum model (a) the third and the fourth mode; (b) the optic and the acoustic mode; 


\section{ACCEPTED MANUSCRIPT}

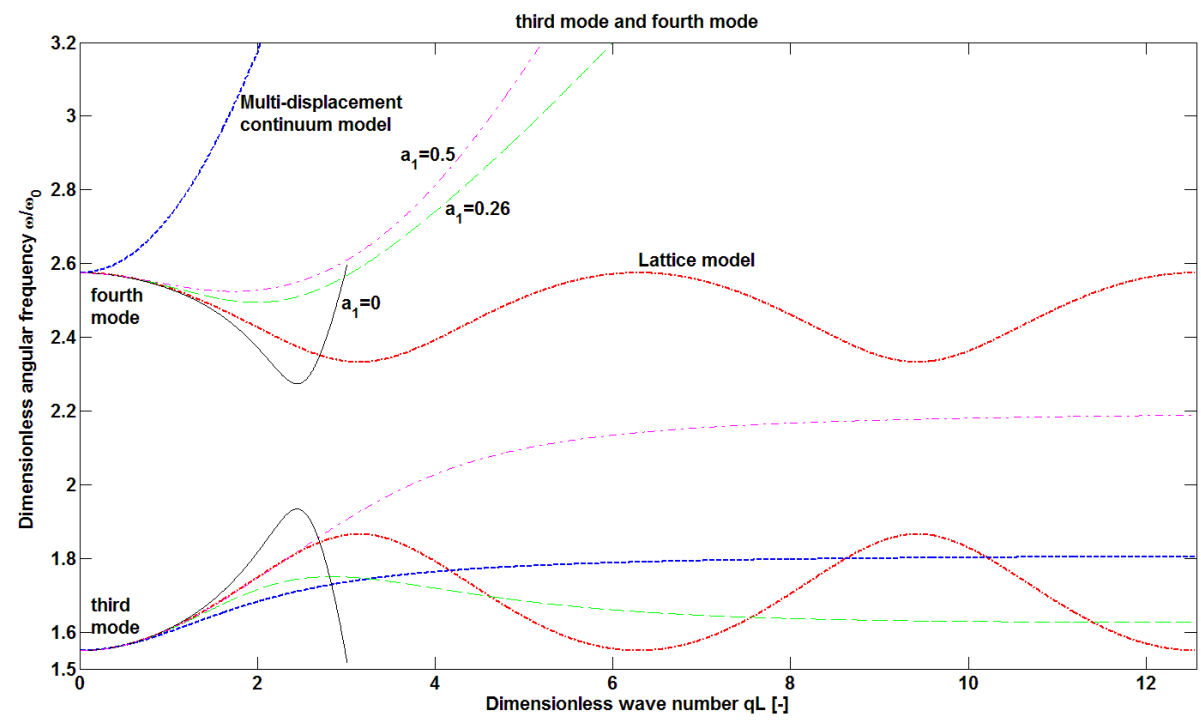

(a)

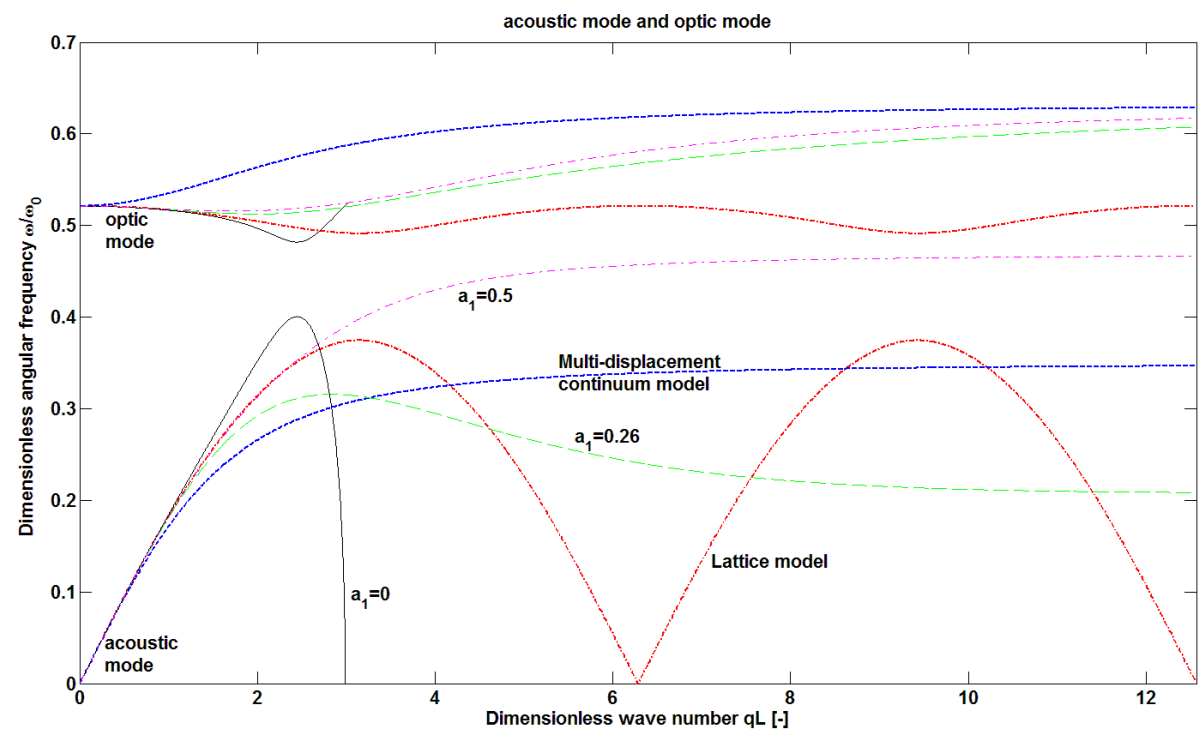

(b)

Fig.9 The dimensionless dispersion curves for the secend third order nonlocal gradient continuum model compared with the metamaterial crystal lattice model and the multi-displacement continuum model (a) the third and the fourth mode; (b) the optic and the acoustic mode; 


\section{ACCEPTED MANUSCRIPT}

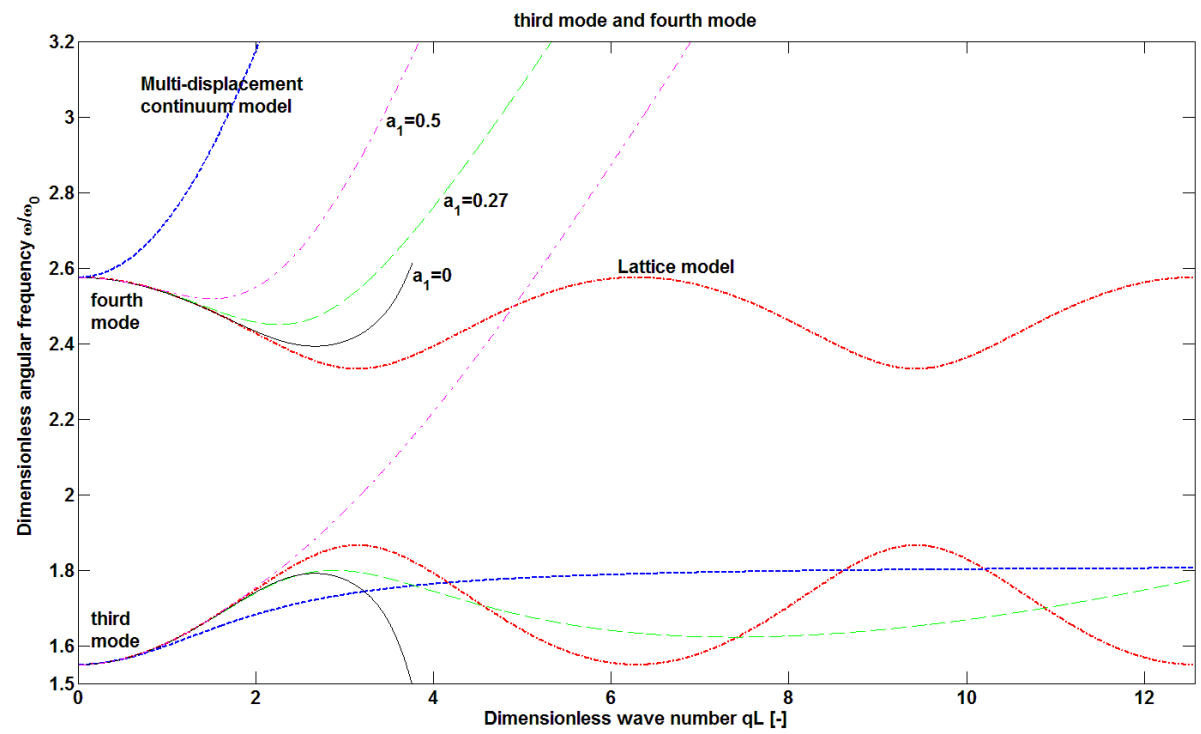

(a)

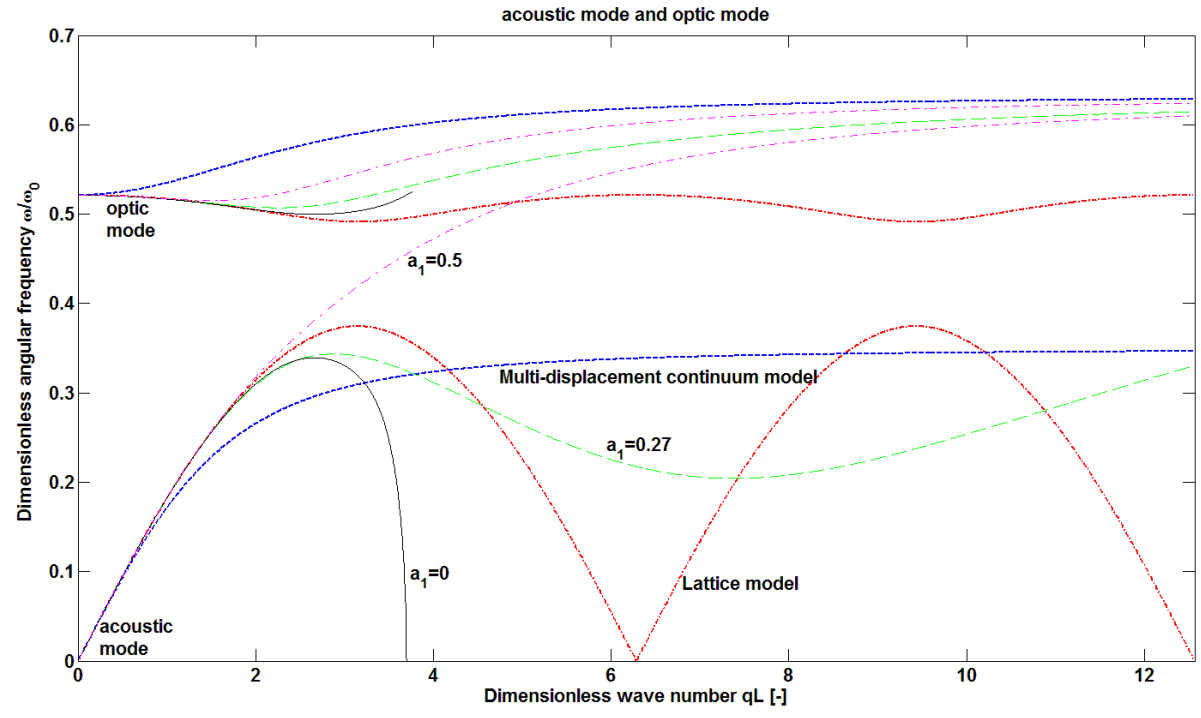

(b)

Fig.10 The dimensionless dispersion curves for the fourth fifth order nonlocal gradient continuum model compared with the metamaterial crystal lattice model and the multi-displacement continuum model (a) the third and the fourth mode; (b) the optic and the acoustic mode; 